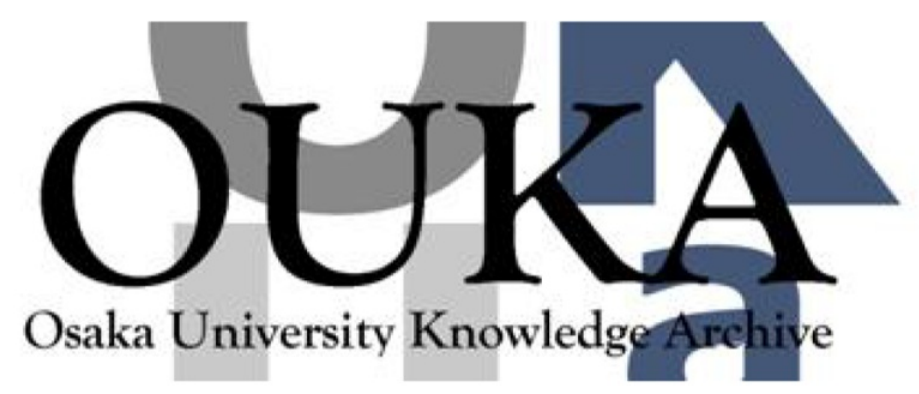

\begin{tabular}{|c|l|}
\hline Title & $\begin{array}{l}\text { Planar shock wave generated by uniform } \\
\text { irradiation from two overlapped partially } \\
\text { coherent laser beams }\end{array}$ \\
\hline Author(s) & Ozaki, N.; Sasatani, Y.; Kishida, K. et al. \\
\hline Citation & Journal of Applied Physics. 89(5) p. 2571-p. 2575 \\
\hline Issue Date & $2001-03-01$ \\
\hline oaire:version & VoR \\
\hline URL & https://hdl. handle.net/11094/3230 \\
\hline rights & \\
\hline Note & \\
\hline
\end{tabular}

Osaka University Knowledge Archive : OUKA

https://ir. Library. osaka-u. ac. jp/

Osaka University 


\title{
Planar shock wave generated by uniform irradiation from two overlapped partially coherent laser beams
}

\author{
N. Ozaki, ${ }^{a)}$ Y. Sasatani, K. Kishida, M. Nakano, M. Miyanaga, K. Nagai, K. Nishihara, \\ T. Norimatsu, and K. A. Tanaka \\ Faculty of Engineering, Osaka University 2-1, Yamada-oka, Osaka 565-0871, Japan and Institute of Laser \\ Engineering, Osaka University 2-6, Yamada-oka, Suita, Osaka 565-0871, Japan \\ Y. Fujimoto, K. Wakabayashi, S. Hattori, T. Tange, and K. Kondo \\ Materials and Structures Laboratory, Tokyo Institute of Technology 4259, Nagatsudacho, \\ Midori, Yokohama, Kanagawa 226-0026, Japan \\ M. Yoshida \\ National Institute of Materials and Chemical Research 1-1, Higashi, Tsukuba, Ibaragi 305-0046, Japan \\ N. Kozu, \\ School of Engineering, University of Tokyo 7-3-1, Hongo, Bunkyo-ku, Tokyo 113-8656, Japan \\ M. Ishiguchi \\ Department of Safety Engineering, Yokohama National University 79-5, Tokiwadai, \\ Hodogaya-ku, Yokohama,Kanagawa 240-8501, Japan \\ H. Takenaka \\ NTT Advanced Technology Company 3-9-11, Midoricho, Musashino, Tokyo 180-0012, Japan
}

(Received 1 December 1999; accepted for publication 28 November 2000)

\begin{abstract}
Two partially coherent light laser beams, coupled with a random phase plate were focused at an angle of $31.7^{\circ}$ with the centers of the beams offset by $250 \mu \mathrm{m}$. This produced a relatively uniform (7\% root-mean-square) irradiated spot of $400 \mu \mathrm{m}$. When this technique was used to produce a shock wave in a copper wedge, a relatively uniform shock of $2 \mathrm{~ns}$ duration was produced. In addition, a multilayered flyer plate was accelerated using this method to an average speed of $21 \mathrm{~km} / \mathrm{s}$ and produced a planar impact on a glass window. It is anticipated that this technique can be used to produce important multi-Mbar equation of state information in future experiments. (C) 2001 American Institute of Physics. [DOI: 10.1063/1.1342189]
\end{abstract}

\section{INTRODUCTION}

The high pressure (and high density) equation of state (EOS) of materials play an important role in the studies of astrophysics, inertial confinement fusion, and other related fields. Recently, high power pulsed lasers have been used to achieve multi-Mbar pressures. ${ }^{1-4}$ However, there are several problems that complicate the laser-driven EOS experiments. One of the main problems is the uniformity and the planarity of shock wave generated; both are determined by the nature of laser itself. A nonuniform irradiation caused by the laser light coherence prevents the generation of a uniform shock. Such irradiation may cause nonlinear corona instabilities which produce hot electrons; this could be the source of target preheat.

Additionally, the shock front must be planar and uniform across the sample materials and the driver material to produce a valid shock experiment that yields useful EOS information. Another concern is the temporal steadiness of the shock pulse. A shock pulse long enough to produce a constant sate during the experiment is required to allow for proper interpretation of the data. In the case of laser experiments, this may be for only a few nanoseconds.

a)Electronic mail: nozaki@ile.osaku-u.ac.jp
We have applied a new laser irradiation method that produced a uniform laser irradiation of a target or flyer plate. Two beams of partially coherent light (PCL) were coupled with a random phase plate (RPP) $)^{5}$ to produce uniform drive. The two focal spots of the PCL laser beams were slightly offset in space. The spatial and temporal characteristics of the shock wave produced by this technique were verified using a wedge target. The shock wave interacting with the wedge face produced a linear relation in both time and space, indicating a steady $400 \mu \mathrm{m}$ diameter shock with a $2 \mathrm{~ns}$ duration. This technique was also applied to multi-layered flyer acceleration. The flyer was extremely flat and achieved an ultrahigh speed.

\section{EXPERIMENT}

The overlapping laser beam setup is shown in Fig. 1. The work was conducted at the GEKKO XII: Nd glass laser system of the Institute of Laser Engineering (ILE), Osaka University. ${ }^{6}$ Two of 12 GEKKO XII laser beams were used and were synchronized within 25 ps. Each laser beam delivered an energy up to $300 \mathrm{~J}$ at a wavelength of $\lambda_{L}=527 \mathrm{~nm}$. The pulse shape was approximately square with a full width half maximum (FWHM) time of $2 \mathrm{~ns}$ and a rise time of 100 ps. 


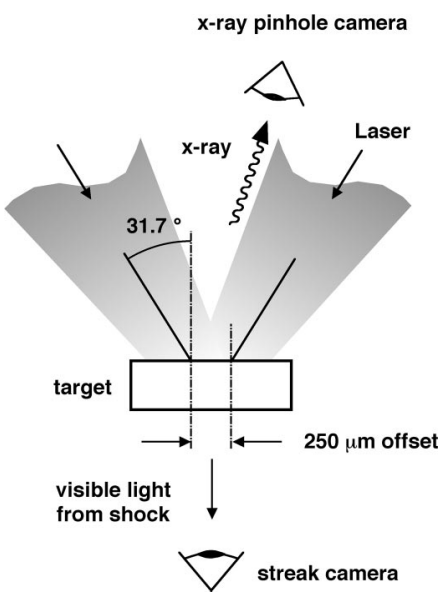

FIG. 1. Diagram of laser irradiation experimental setup. Two PCL beams with Gaussian focus patterns irradiate a target at the same time with a slight offset $(250 \mu \mathrm{m})$. The laser wavelength is $527 \mathrm{~nm}$, the pulse duration is $2 \mathrm{~ns}$ (FWHM).

The laser light was optically modified so that it was partially coherent and a RPP was used to produce a more uniform beam. A RPP was used to segment the large aperture laser beam into a large number of small beamlets and thereby greatly reduced the irradiation nonuniformity. However, the focused beam pattern through the RPP produces fine interference speckle structures which depend on the coherence of the incident light. If the coherence was not partially destroyed, the speckle structures would be imprinted on the target as surface irregularities and would produce Rayleigh-Taylor instabilities. This problem was improved by using PCL which causes both spatial and temporal incoherence in the beam. This principle of beam smoothing is based on the short coherence time and produces the rapid change of the focused beam pattern to smooth out short wavelength speckles. PCL has achieved a uniform beam (only $2 \%$ nonuniformity) with an averaging time of $100-200$ ps. $^{5}$

In our experiment, these modified beams irradiated the target at an incident angle of $31.7^{\circ}$ (this angle was constrained by the size and shape of the target chamber). The two simultaneous beams were focussed on a target surface with the centers offset laterally by $250 \mu \mathrm{m}$. The focal spot diameter of each beam was $500 \mu \mathrm{m}$ (FWHM) at the target. Although the intensity distribution of each beam focal spot is a Gaussian profile, the beam offset allows for partial overlap of the two Gaussian profiles, producing a flat-topped area in the overlapped region. This area was used to irradiate the targets in the experiments and produced a uniform shock drive.

\section{RESULTS AND DISCUSSION}

\section{A. Irradiation pattern of the overlapped beams}

An x-ray pinhole camera, coupled with a charge coupled device $(\mathrm{CCD})$ detector (16 bits, $1152 \times 1242$ pixels) was used to monitor the irradiation pattern of the overlapped beams as shown in Fig. 1. The magnification of the pinhole camera was $M=8.6$ with a pinhole diameter of $12 \mu \mathrm{m}$. A $12 \mu \mathrm{m}$ thick aluminum filter was placed in front of the x-ray CCD.

In the experiments, emission at the rear surface of target caused by the sock wave breakout was recorded with an electronic streak camera system clotted outside the vacuum chamber. The signals were collected by an $f / 1.4$ lens and were image relayed to the streak camera which was coupled to a CCD detector ( 16 bits, $512 \times 512$ pixels). The detectable wavelength of this streak camera is between 200 and $850 \mathrm{~nm}$.

Figure 2(a) is a typical image taken using the $x$-ray pinhole camera. Energies of the two PCL beams were $221 \mathrm{~J}$ and 228 J. In Fig. 2(a), the white line $\left(A-A^{\prime}\right)$ shows the position of the streak camera slit. An intensity scan along the $A-A^{\prime}$ line is shown in Fig. 2(b). This profile indicates that overlapping the two Gaussian beams produces a flat region in the intensity along line $A-A^{\prime}$. The darker part of the profile defines the region about $80 \%$ of the maximum signal on both sides. The nonuniformity of the flat area is $7 \%$ root-meansquare (rms) over the $400 \mu \mathrm{m}$ lien defined by the $80 \%$ points. This level of uniformity is much better than that for other laser spots developed using only optical smoothing. ${ }^{7}$ Because of the low signal to noise ratio due to rather heavy filtration in the $\mathrm{x}$-ray picture, the actual beam uniformity might be worse than has been shown here by about $5 \%$.

\section{B. Spatial and temporal characteristics of a generated shock wave}

An experiment was performed to determine the spatial and temporal characteristics of the shock wave driven by this type of beam profile. A copper wedge target was used with a $5.7^{\circ}$ angle; it had $35 \mu \mathrm{m}$ rise over $350 \mu \mathrm{m}$ as shown in Fig. 3 . The surface of the wedge target was optically polished to a flatness of a few thousand $\AA$ rms. If the laser pulsed launches a planar shock wave with a constant state behind it in the wedge, the breakout profile on the opposite side of the wedge should be linear along the wedge. The bottom part of Fig. 3 shows the streak camera image. The upper part of the image is a time fiducial from the drive beam; it had a $2 \mathrm{~ns}$ pulse duration (FWHM). Energies for the two beams in this experiment were 218 and $210 \mathrm{~J}$, corresponding to an irradiance of $1.4 \times 10^{14} \mathrm{~W} / \mathrm{cm}^{2}$. The lower part of the image is from recording the emission produced by the shock wave as it breaks out from the wedge; it is roughly a straight line with a slope. In Fig. 4, the fiducial and the shock breakout profiles are plotted. Time histories of the emission are also shown at positions of 40,80 , and $120 \mu \mathrm{m}$ and the solid diamonds indicate the position that is plotted on the time-distance diagram. The solid squares and the solid diamond correspond to the fiducial start and the front part of the shock breakout, respectively. Time errors in the shock breakout are estimated to be $\pm 9 \%$ or less due to the time resolution of the streak camera. Shock velocity in the copper wedge was determined using the wedge thickness and the time from fiducial start to shock breakout; the velocity was $10.7 \pm 0.9 \mathrm{kms} / \mathrm{s}$. Using the copper EOS, this corresponds to a pressure in the wedge of $\sim 3.5$ Mbar.

Decay in the breakout emission occurs at a distance of about $150 \mu \mathrm{m}$ along the wedge in Fig. 3 so the line in Fig. 4 


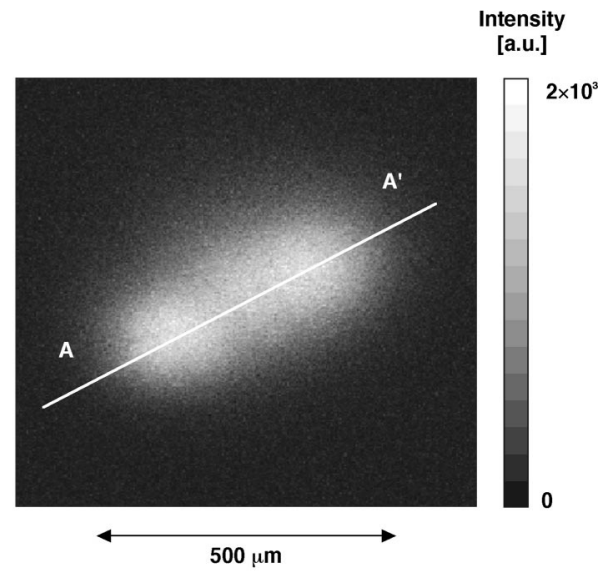

(b)

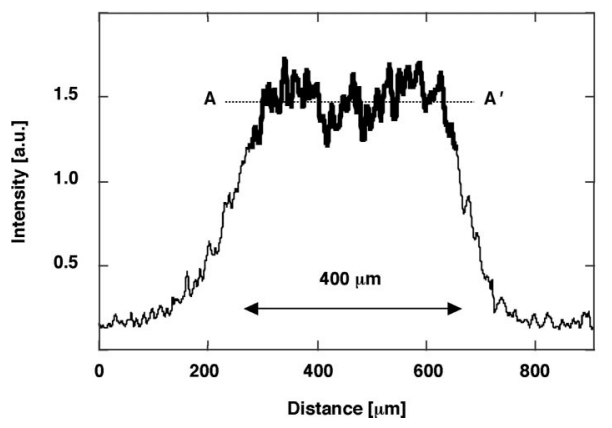

FIG. 2. Image from $x$-ray pinhole camera when the target is irradiated is shown in (a). The intensity profile along the white line between $A$ and $A^{\prime}$ is shown in (b). The dotted horizontal line is the intensity average of the part of the thick solid line (at the $80 \%$ points) in the profile. The width of the thick solid line is $400 \mu \mathrm{m}$ and the uniformity is within $7 \%$ (rms) from the average.

was stopped at this point. This is an indication that the shock-pulse trailing rarefaction catches up with the front at this point and begins to attenuate the shock pulse. The positions of 0 and $150 \mu \mathrm{m}$ on the base of the wedge correspond to 30 and $45 \mu \mathrm{m}$ thickness in the wedge at those points,

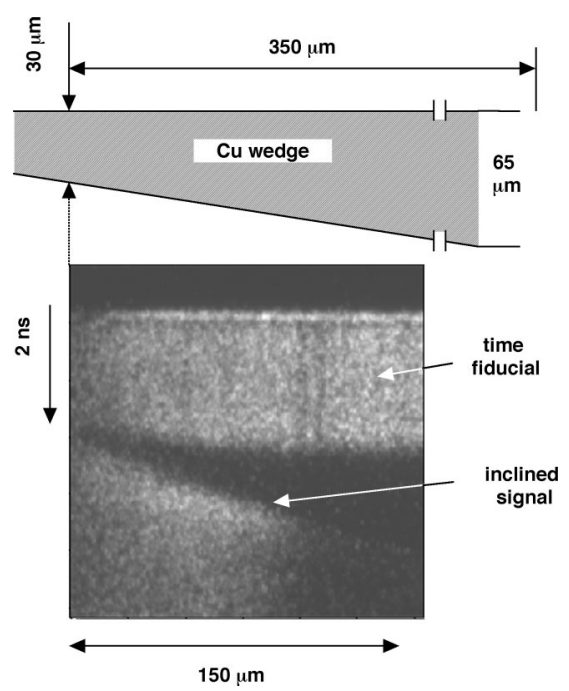

FIG. 3. Diagram of copper wedge experiment and the streak image obtained from the experiment. The wedge target is $30 \mu \mathrm{m}$ at the thinnest and $65 \mu \mathrm{m}$ at the thickest position $350 \mu \mathrm{m}$ away. The time fiducial and the shock breakout signal are shown.

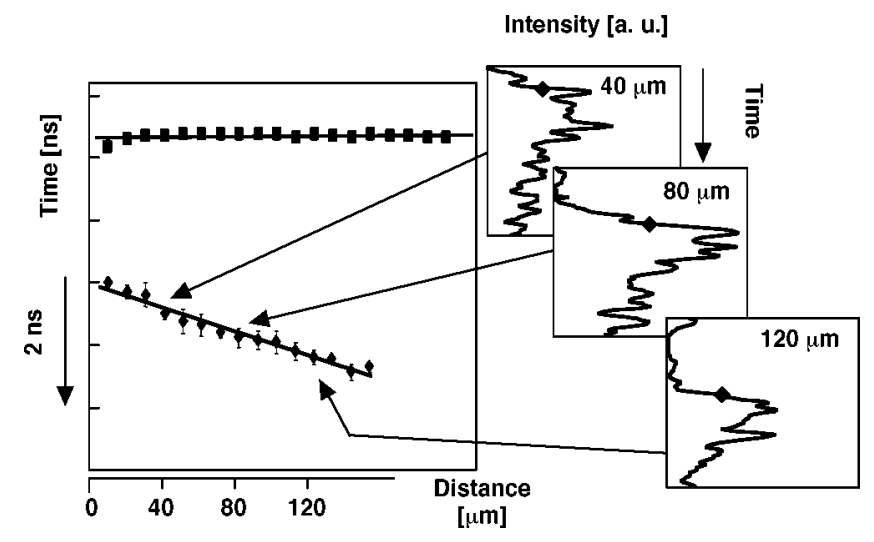

FIG. 4. Shock breakout timing is plotted as a function of position from the streak record in Fig. 3. Solid diamonds and squares correspond to the shock breakout and the time fiducial. The time histories of the emission are also shown at 40,80 , and $120 \mu \mathrm{m}$ to give an idea of how the breakout positions were determined.

respectively. We did a one-dimensional (1-D) Lagrangian hydrodynamic simulation ${ }^{8}$ to see if this catch up position was reasonable. The time-distance diagram produced from this calculation is shown in Fig. 5. Inputs for the calculation were that an initial shock pulse of 3.5 Mbar and 2 ns duration was introduced to a piece of copper at a free surface and then the problem was run to see at what copper thickness the rarefaction catches up to the shock wave front. Figure 5 consists of several zones; zone 1 is unshocked $\mathrm{Cu}$, zone 2 is shocked $\mathrm{Cu}$, and zone 3 is the area where the rarefaction propagates. The shock front and the rarefaction front are straight lines, respectively. This simulation code uses the SESAME EOS database $^{9}$ for the copper EOS. Figure 4 shows that the head of the rarefaction catches up with the shock front at a thickness of about $4 \mathrm{t} \mu \mathrm{m}$. This corresponds nicely with the $45 \mu \mathrm{m}$ thickness of the wedge at the $150 \mu \mathrm{m}$ position in the experiment, indicating that the interpretation given above is reasonable. This simulation was used to provide the estimate that the laser driven shock pulse has a duration of about 2 ns.

\section{Application to the laser flyer}

In addition to directly driving a shock wave into a sample with laser deposition, a rectangular-shaped shock pulse can be produced by using the laser to accelerate a flyer plate which then impacts the target If the flyer plate can be laser driven so that it is planar and its velocity is well known, a well understood shock pulse in the target material is developed. In addition, laser accelerated flyers may possibly avoid the preheat effect due to X-ray and/or hot electrons, one of the critical problems in laser direct-drive EOS experiments. ${ }^{2,10}$ Conventional methods to produce planar flyers of relatively large size use gas guns or explosive planewave generators to accelerate them. These methods produce EOS measurements with $1 \%$ to $2 \%$ accuracy but the flyer speeds are limited to about $8 \mathrm{~km} / \mathrm{s}$ and the achievable pressures to a few Mbars. We have previously demonstrated that a $20 \mathrm{~J}$ class laser can drive flyers up to $10 \mathrm{~km} / \mathrm{s}^{11}$

We have used the laser irradiation technique to accelerate a flyer plate. The flyer had a multilayered structure to minimize the mass so that higher speeds could be attained 


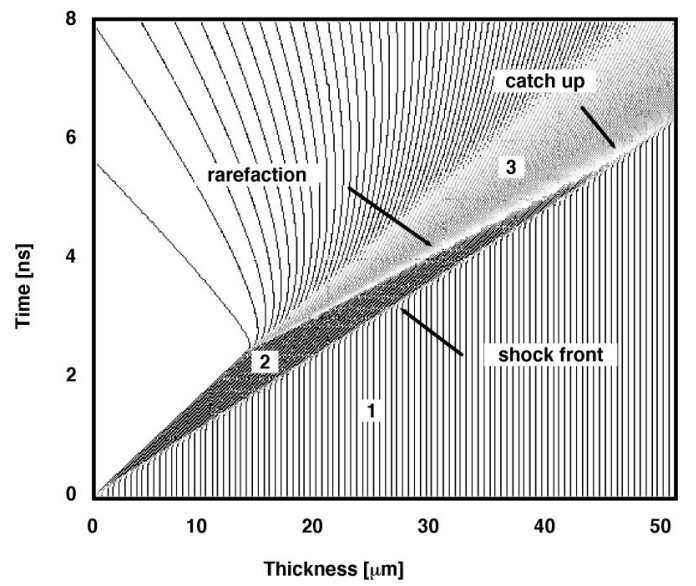

FIG. 5. Propagation of the shock and rarefaction wave calculated by a 1D hydrodynamic code simulation: zone 1 is the unshocked copper; zone 2 is the shocked copper; zone 3 is the region where the rarefaction propagates. The laser intensity is $1.0 \times 10^{14} \mathrm{~W} / \mathrm{cm}^{2}$. The duration of laser pulse is $2 \mathrm{~ns}$ (FWHM). The head of the rarefaction catches up with the shock front at a thickness of about $45 \mu \mathrm{m}$.

than with a single material flyer. The multilayered consisted of aluminum $(\mathrm{Al})$, polyimide (PI), and tantalum $(\mathrm{Ta})$ each with a thickness of $2 \mu \mathrm{m}, 150 \mu \mathrm{m}$, and $2 \mu \mathrm{m}$, respectively. The PCL beams irradiated the Al side. The Al layer works as a shine through shield to block the low intensity part of a laser pulse and a plasma is formed in it. The $\mathrm{Al}$ and perhaps some of the PI acts as an ablator. The PI/Ta layers then become the flyer that is accelerated.

It is desirable to have the flyer remain in a condensed state during the acceleration and flight. Ta was selected as the flyer material because it has a high boiling point $(5400$ $\mathrm{K})$. Because the PI is thick compared to the Ta, shock reverberation occur in the Ta that increase its speed over what it would be if it were pure Ta. The details of the acceleration mechanism are discussed in Ref. 11.

Figure 6 shows the experimental setup for the flyer experiment. A streak camera was used to record the emission that occurs when the flyer streaks the Al coated glass window; Fig. 6(b) presents the streak image obtained. The upper signal in the streak image is the time fiducial. The glass window target had a $65 \mu \mathrm{m}$ step which was used to determine the flyer velocity. The Al coating on the window was $0.2 \mu \mathrm{m}$ thick and stopped stray light from entering the streak camera. Energies of the two beams were 224 and $228 \mathrm{~J}$. Using the time between the emission at the two level and step height, the average flyer velocity was estimated to be 21 $\mathrm{km} / \mathrm{s}$, a very high speed. In addition to being a high speed, the straight emission line in Fig. 6(b) indicates that the flyer was quite flat at the time it impacted the glass window.

For a comparison, a streak image of a laser-accelerated flyer impacting a similar glass target from previous work is shown in Fig. 6(c). This streak image was obtained using a laser, without any beam smoothing, to accelerate the flyer. The laser wavelength was $1053 \mathrm{~nm}$ and the intensity was $1.4 \times 10^{13} \mathrm{~W} / \mathrm{cm}^{2}$. In this experiment, the multilayered flyer was composed of $0.2 \mu \mathrm{m} \mathrm{Al}, 20 \mu \mathrm{m} \mathrm{PI}$, and $1 \mu \mathrm{m} \mathrm{Ta}$. The streak was obtained in the same way except the glass window had a step of $150 \mu \mathrm{m}$. In this experiment, the flyer (a)

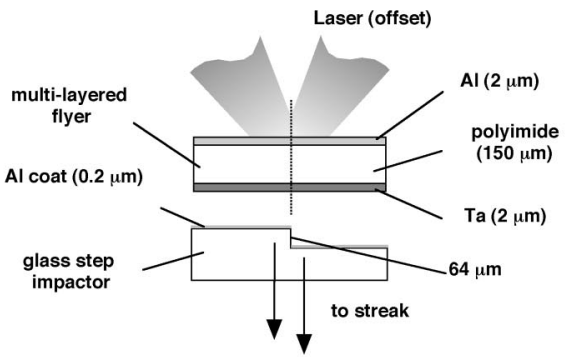

(b)

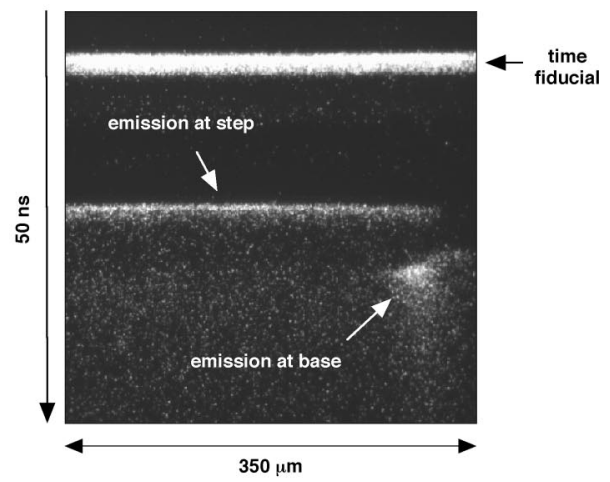

(c)

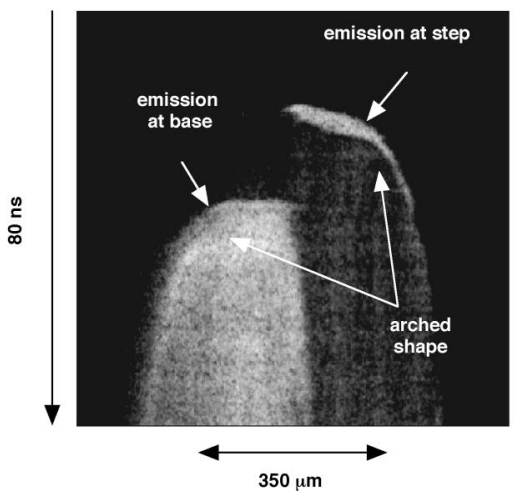

FIG. 6. Diagrams of multilayered flyer and glass step target experiment are shown in (a). The streak camera record of the experiment is shown in (b). The record of the flyer hitting the glass window shows a straight line indicating a flat flyer for the drive method described in this article. For comparison purposes, the streak record from a similar experiment with a conventional laser drive with only one laser beam is shown in (c). Clearly the flyer had a arched shape and was not near as flat as the flyer shown in (b).

velocity was $7.9 \mathrm{~km} / \mathrm{s}$. The Gaussian shaped pulse of the driving laser obviously caused the flyer to have an arched shape. Obviously the flyer which produced the streak in Fig. $6(\mathrm{~b})$ is much flatter than the one producing the streak shown in Fig. 6(c).

\section{CONCLUSIONS}

The technique of using two PCL beams coupled with RPP and focussed at an angle with each other and offset (as described in this paper) produced a much improved planar and uniform drive. The nonuniformity of the $400 \mu \mathrm{m}$ unusable area of the beam was only $7 \%$ (rms). A copper wedge experiment verified that direct shock wave drive was relatively planar and had a duration of about 2 ns. A multilayer 
flyer acceleration experiment showed that the flyer achieved a high speed of $21 \mathrm{~km} / \mathrm{s}$ and produced a flyer that was quite flat.

\section{ACKNOWLEDGMENTS}

This work was supported by CREST (Core Research for Evolutional Science and Technology) program of Japan Science and Technology Corporation (JST). The authors gratefully acknowledge the valuable support for the experiment by GEKKO XII technical crews and scientists at ILE. Especially, the authors would like to thank N. Morio, S. Urushihara, K. Suzuki, and T. Kawasaki for operating the GEKKO XII, O. Maegawa, K. Shimada, T. Komeno, T. Syozaki, H. Azuma, and M. Oshikawa for the diagnostic support, Y. Kimura for target fabrication, and Dr. R. Kodama for useful discussions concerning the experiment.

${ }^{1}$ L. B. DaSilva et al., Phys. Rev. Lett. 78, 483 (1997).

${ }^{2}$ R. Cauble et al., Phys. Plasmas 4, 1857 (1997).
${ }^{3}$ Sizu Fu, Yuan Gu, Jiang Wu, and Shiji Wang, Phys. Plasmas 2, 3461 (1995).

${ }^{4}$ R. Cauble, D. W. Phillion, T. J. Hoover, N. C. Holmes, J. D. Kikenny, and R. W. Lee, Phys. Rev. Lett. 70, 2102 (1993).

${ }^{5}$ H. Nakano, K. Tsubakimoto, N. Miyanaga, M. Nakatsuka, T. Kanabe, H. Azechi, T. Jitsuno, and S. Nakai, J. Appl. Phys. 73, 2122 (1993).

${ }^{6} \mathrm{C}$. Yamanaka, in Proceedings of the Inertial Fusion Science and Applications '99, Bordeaux, France, September, 1999, pp. 19-31.

${ }^{7}$ D. Batani, S. Bossi, A. Benuzzi, M. Koenig, B. Faral, J. M. Boudenne, N. Grandjouan, S. Atzeni, and M. Temporal, Laser Part. Beams 14, 211 (1996).

${ }^{8}$ M. Yoshida, "Program MYIDL One Dimensional Lagrangian Hydrodynamic Code," CETR Report C-06-86, Center for Explosives Technology Research (1986).

${ }^{9}$ See National Technical Information Service Document No. DE85011902 (Los Alamos National Laboratory Report LA-10160-MS, by K. Holion, 1984). Copies may be ordered from the National Technical Information Service, Springfield, Virginia 22161.

${ }^{10}$ A. Benuzzi, M. Koenig, B. Raral, J. Krishnan, F. Pisani, T. Hall, S. Ellwi, S. Huller, J. Honrubia, and N. Grandjouan, Phys. Plasmas 5, 1 (1998).

${ }^{11}$ A. Tanaka, M. Hara, N. Ozaki, Y. Sasatani, K. Kondo, M. Nakano, K. Nishihara, H. Takenaka, M. Yoshida, and K. Mima, Phys. Plasmas 7, 676 (2000). 